\title{
Uso de substâncias lícitas entre os estudantes de Fonoaudiologia de uma universidade pública*
}

\author{
Nathalia Gama Puppim ${ }^{1}$ \\ (D) https://orcid.org/0000-0002-7705-6438 \\ Flávia Batista Portugal ${ }^{2}$ \\ (D) https://orcid.org/0000-0002-4425-2627 \\ Marluce Mechelli de Siqueira² \\ (D) https://orcid.org/0000-0002-6706-5015
}

\footnotetext{
Artigo extraído da dissertação de mestrado "Uso de álcool e tabaco entre estudantes de Fonoaudiologia de uma universidade pública ", apresentada à Universidade Federal do Espírito Santo, Vitória, ES, Brasil.

${ }^{1}$ Universidade Federal do Espírito Santo, Hospital Universitario Cassiano Antônio Moraes Vitória, ES, Brasil.

2 Universidade Federal do Espírito Santo, Vitória, ES, Brasil.
}

Objetivo: analisar o consumo de álcool e tabaco entre os estudantes do curso de Fonoaudiologia de uma universidade pública. Método: trata-se de um estudo transversal; a investigação foi fundamentada no preenchimento do instrumento proposto pela Secretaria Nacional de Políticas sobre Drogas. O estudo possui uma amostra de 130 estudantes matriculados no curso de Fonoaudiologia, o que corresponde a 79,06\% dos alunos matriculados em 2018. Resultados: o álcool apresenta-se como uma das substâncias lícitas mais consumidas entre os universitários, o uso na vida, no ano e nos últimos 30 dias foram de 79,2\%, 56,2\% e $45,4 \%$, respectivamente. Quanto ao tabaco, $21,5 \%$ relataram que realizaram o consumo de tabaco em algum momento da vida; no ano, 8,5\% e, nos últimos 30 dias, 7,7\%. Na análise multivariada, as variáveis preditoras significativas para o uso de álcool foram idade (no mês) e prática religiosa (no ano e no mês). Já para o uso do tabaco foram: sexo (ao longo da vida), prática religiosa (ao longo da vida e no ano) e grupo étnico (no ano). Conclusão: os achados deste estudo apontam que prevalece o consumo das substâncias lícitas entre os universitários, tornando-se importante maior abordagem do tema e ações de prevenção ao uso indevido dessas substâncias e de promoção de saúde nesta população.

Descritores: Bebidas Alcoólicas; Uso de Tabaco; Estudantes; Fonoaudiologia; Prevenção.

\section{Como citar este artigo}

Puppim NG, Portugal FB, Siqueira MM. Use of licit substances among Speech Therapy undergraduate students of a public university. SMAD, Rev Eletrônica Saúde Mental Álcool Drog. 2021 abr.-jun.;17(2):16-25. doi: https://dx.doi.org/10.11606/issn.1806-6976.smad.2021.169569 


\section{Use of licit substances among Speech Therapy undergraduate students of a public university}

Objective: analyze the consumption of alcohol and tobacco among Speech Therapy undergraduate students of a public university. Method: this is a cross-sectional study, based on the completion of an instrument proposed by the National Secretariat for Drug Policy. The study analyzed a sample of 130 students enrolled in the Speech Therapy course, which corresponds to $79.06 \%$ of students enrolled in 2018. Results: alcohol is one of the most commonly used legal substances among university students; alcohol consumption rates in life, in the year, and in the last 30 days were: $79.2 \%, 56.2 \%$ and $45.4 \%$, respectively. Regarding tobacco, $21.5 \%$ reported using tobacco at some point in life; $8.5 \%$ in the year, and $7.7 \%$ in the last 30 days. In the multivariate analysis, the significant predictor variables for alcohol consumption were age (in the month) and religious practice (year and month). For tobacco use, the significant predictor variables were: sex (throughout life), religious practice (throughout life and in the year) and ethnic group (in the year). Conclusion: the findings of this study indicate that the consumption of licit substances prevails among university students, highlighting the importance of adopting actions to prevent inadequate use of these substances and promote health among this population.

Descriptors: Alcoholic Beverages; Use of tobacco; Students; Speech Therapy; Prevention.

\section{Uso de sustancias lícitas entre los estudiantes de Fonoaudiología de una universidad pública}

Objetivo: analizar el consumo de alcohol y tabaco entre estudiantes de Fonoaudiología de una universidad pública. Método: estudio transversal; investigación fundamentada en el completado del instrumento propuesto por la Secretaría Nacional de Políticas sobre Drogas. Estudio sobre muestra de 130 estudiantes matriculados en la carrera de Fonoaudiología, equivalentes al 79,06\% del alumnado matriculado en 2018. Resultados: el alcohol se presenta como una de las sustancias lícitas más consumidas entre los estudiantes. Fue consumido en la vida, en el año y en los últimos 30 días por el $79,2 \%, 56,2 \%$ y $45,4 \%$, respectivamente. Acerca del tabaco, el $21,5 \%$ informó haberlo consumido en algún momento de la vida; $8,5 \%$ en el año; y $7,7 \%$ en los últimos 30 días. En el análisis multivariado, las variables predictivas significativas para consumo de alcohol fueron: edad (para el mes) y práctica religiosa (para el año y el mes). Con el tabaco, fueron: sexo (a lo largo de la vida), práctica religiosa (a lo largo de la vida y para el año) y grupo étnico (para el año). Conclusión: los hallazgos muestran prevalencia del consumo de sustancias lícitas entre los universitarios, haciendo que abordaje mayor del tema y acciones de prevención del uso indebido de sustancias y de promoción de salud resulten importantes para esta población.

Descriptores: Bebidas Alcohólicas; Uso del Tabaco; Estudiantes; Terapia del Lenguaje; Prevención. 


\section{Introdução}

O consumo de drogas lícitas tem sido cenário de destaque nas discussões de saúde pública mundial. 0 uso dessas substâncias acarreta implicações negativas na saúde física e mental, individual e coletiva do indivíduo(1). Desse modo, observa-se aumento de impactos importantes na sociedade, tais como: gastos com internações e problemas hospitalares, problemas sociais, diminuição do desempenho acadêmico, déficits em desenvolvimento e habilidades cognitivas, aumento de indicadores de acidentes do trânsito, violências e homicídios ${ }^{(2-4)}$.

O uso das drogas lícitas tem-se tornado causador de várias doenças e agravos no mundo. Estudos mostram que a ingestão prolongada do álcool e tabaco acarreta patologias vocais como pólipos, nódulos, laringite aguda e carcinoma laríngeo. Em razão da exposição ao tabaco, existem casos de edema e doenças benignas na laringe, e ao álcool, aumento do risco para neoplasias de laringe ${ }^{(4-5)}$.

O crescimento do consumo do álcool e tabaco entre jovens e a idade de experimentação cada vez mais precoce são fatores preocupantes, pelo fato de se aumentar o risco de serem adultos que bebam e fumem ${ }^{(5)}$. Nesse contexto, pesquisas nacionais identificaram que o consumo dessas substâncias destaca-se no cenário dos estudantes universitários ${ }^{(6-8)}$. Existem evidências de que o consumo de álcool é maior entre os universitários, quando comparado ao da população geral(5).

$O$ ingresso na universidade caracteriza-se como uma nova etapa na vida dos estudantes. A entrada no curso superior desperta curiosidade e autonomia, surgem os encontros sociais, favorecendo, em algumas situações, o consumo de álcool e tabaco(7).

Os universitários da área da saúde, em especial os alunos de Fonoaudiologia, serão futuros profissionais que levarão noções básicas de saúde à comunidade. 0 uso dessas substâncias é agressivo ao mecanismo vocal. É de extrema importância que o fonoaudiólogo tenha conhecimento das possíveis alterações causadas pelo uso das substâncias lícitas. Diante da complexidade do tema, os estudos realizados com usuários de drogas da área de voz ainda são limitados e escassos ${ }^{(9)}$. O objetivo desse estudo é analisar o consumo de álcool e tabaco entre estudantes da Fonoaudiologia de uma universidade pública.

\section{Método}

Trata-se de um estudo transversal realizado no período de fevereiro a julho de 2018. Participaram do estudo 136 alunos matriculados do primeiro ao último período do curso de Fonoaudiologia de uma universidade pública. Os critérios de inclusão foram: ser estudante com idade igual ou superior a 18 anos e devidamente matriculado no ano de 2018. Entretanto, 6 questionários foram excluídos por constar preenchimento inadequado do instrumento da pesquisa, resultando em uma amostra de 130 questionários.

O instrumento utilizado foi o questionário proposto pela Secretaria Nacional de Políticas sobre Drogas (SENAD) para o I Levantamento Nacional sobre o Uso de Álcool e Outras Drogas entre Universitários das 27 capitais brasileiras, realizado em $2009^{(10)}$. O instrumento de pesquisa é dividido em seções, com a proposta de coletar dados socioeconômicos e demográficos, informações acadêmicas, atividades gerais, satisfação e desempenho acadêmico, consumo geral de drogas, consumo de tabaco e derivados, consumo de álcool, detalhamento do consumo de outras drogas e comportamentos gerais ${ }^{(10)}$.

Para análise dos dados, utilizou-se o programa estatístico adequado. Primeiramente, foi realizada uma análise univariada para descrição das variáveis qualitativas, representadas pela frequência absoluta simples $(\mathrm{N})$ e frequência relativa simples (\%). Posteriormente, foi realizada a análise bivariada para verificar a associação das variáveis desfecho "Uso de álcool na vida", "Uso de álcool no ano", "Uso de álcool no mês" e "Uso de tabaco na vida", "Uso de tabaco no ano", "Uso de tabaco no mês", utilizando-se o teste Qui-quadrado como alternativa quando a amostra era pequena ou, quando $80 \%$ dos valores esperados eram inferiores a 5, aplicou-se o teste exato de Fisher; consideraram-se estatisticamente significativas diferenças com $p<0,05$. Por fim, realizou-se uma análise multivariada por meio da regressão logística múltipla, calcularam-se sua respectiva razão de chances (OR - sigla do inglês) e seus Intervalos de Confiança (IC), na qual foram selecionadas, para o modelo, somente as variáveis associadas ao desfecho, com um nível de significância $p<0,100$ na análise bivariada.

O presente estudo foi aprovado ( $n^{0}$ 1.566.208) pelo Comitê de Ética em Pesquisa (CEP) do Centro de Ciências da Saúde (CCS) da Universidade Federal do Espírito Santo (UFES), sendo conduzido de acordo com a Resolução No 466/2012 do Conselho Nacional de Saúde(11). Todos os indivíduos envolvidos assinaram o Termo de Consentimento Livre e Esclarecido.

\section{Resultados}

Da amostra de 172 estudantes matriculados no curso de Fonoaudiologia de uma universidade pública, excluíram-se 36 (20,93\%) por serem menores de 18 anos e $6(3,48 \%)$ por preenchimento inadequado do instrumento da pesquisa. Participaram do estudo 130 estudantes, o que corresponde a 79,06\% dos alunos.

A média de idade dos estudantes foi de 21,54 anos $(D P=3,47)$, com faixa etária mínima de 18 e máxima de 38 anos. Houve predomínio de estudantes do sexo feminino $(93,8 \%)$, solteiros $(96,2 \%)$, residentes com pais/ 
cônjuge/namorado ou filhos (73,8\%), com uma religião $(86,2 \%)$, praticante da religião da qual eram adeptos $(77,7 \%)$, sem filhos $(97,7 \%)$ e sem atividade remunerada $(82,3 \%)$. Verificou-se que $71(54,6 \%)$ dos estudantes se declararam como negros/mulatos ou pardos e 76 (58,5\%), em relação à classe socioeconômica, inseriram-se nos estratos C/D (Tabela 1).

Tabela 1 - Perfil socioeconômico e demográfico dos estudantes de Fonoaudiologia de uma universidade pública. Vitória, ES, Brasil, 2019

\begin{tabular}{|c|c|c|}
\hline Variáveis & $\mathbf{n}$ & $\%$ \\
\hline \multicolumn{3}{|l|}{ Sexo } \\
\hline Masculino & 8 & 6,2 \\
\hline Feminino & 122 & 93,8 \\
\hline \multicolumn{3}{|l|}{ Estado civil } \\
\hline Solteiro / Separado / Divorciado & 125 & 96,2 \\
\hline Casado / Vive junto & 5 & 3,8 \\
\hline \multicolumn{3}{|l|}{ Grupo étnico } \\
\hline Branco & 59 & 45,4 \\
\hline Negro / Mulato / Pardo & 71 & 54,6 \\
\hline \multicolumn{3}{|l|}{ Religião } \\
\hline Não tenho religião & 18 & 13,8 \\
\hline Tenho religião & 112 & 86,2 \\
\hline \multicolumn{3}{|l|}{ Prática religiosa } \\
\hline Pratica religião (Sim) & 101 & 77,7 \\
\hline Não pratica (Não) & 29 & 22,3 \\
\hline \multicolumn{3}{|l|}{ Você tem filhos? } \\
\hline Sim & 3 & 2,3 \\
\hline Não & 127 & 97,7 \\
\hline \multicolumn{3}{|l|}{ Reside com quem? } \\
\hline Sozinho / Amigos / Moradia estudantil / Outros & 34 & 26,2 \\
\hline Pais / Cônjuge / Namorado / Filhos & 96 & 73,8 \\
\hline \multicolumn{3}{|l|}{ Atividade remunerada } \\
\hline Sim & 23 & 17,7 \\
\hline Não & 107 & 82,3 \\
\hline \multicolumn{3}{|l|}{ Classe Econômica } \\
\hline Classe A/B & 54 & 41,5 \\
\hline Classe $C / D$ & 76 & 58,5 \\
\hline Total & 130 & 100,0 \\
\hline
\end{tabular}

Quanto aos padrões de uso de álcool e tabaco apresentados pelos universitários, observou-se que 103 $(79,2 \%)$ dos universitários referiram ter feito uso de álcool na vida, e $73(56,2 \%)$ uso no ano e $59(45,4 \%)$ nos últimos 30 dias que antecederam a pesquisa. Verificou-se que, em relação ao consumo de tabaco e derivados, 28 $(21,5 \%)$ relataram já ter feito uso alguma vez na vida, $11(8,5 \%)$, no ano e $10(7,7 \%)$, no mês (Tabela 2$)$.

Tabela 2 - Padrões de uso (na vida, no ano e no mês) de álcool e tabaco entre estudantes de Fonoaudiologia de uma universidade pública. Vitória, ES, Brasil, 2019

\begin{tabular}{lcccc}
\hline \multirow{2}{*}{ Padrões } & \multicolumn{2}{c}{ Álcool } & \multicolumn{2}{c}{ Tabaco } \\
\cline { 2 - 5 } & $\mathbf{n}$ & $\%$ & $\mathbf{n}$ & $\%$ \\
\hline Uso na vida & & & & \\
Sim & 103 & 79,2 & 28 & 21,5 \\
Não & 27 & 20,8 & 102 & 78,5 \\
\hline Uso no ano & & & & \\
Sim & 73 & 56,2 & 11 & 8,5 \\
Não & 57 & 43,8 & 119 & 91,5 \\
\hline Uso no mês & & & & \\
Sim & 59 & 45,4 & 10 & 7,7 \\
Não & 71 & 54,6 & 120 & 92,3 \\
$\quad$ Total & 130 & 100,0 & 130 & 100,0 \\
\hline
\end{tabular}

Na análise bivariada, foi encontrada relação estatisticamente significativa das variáveis desfecho "Uso de álcool na vida, no ano e no mês" e "Uso de tabaco e derivados na vida, no ano e no mês" somente com a "prática religiosa" (Tabelas 3 e 4).

Tabela 3 - Fatores associados ao uso de álcool entre estudantes de Fonoaudiologia de uma universidade pública, segundo o uso na vida, no mês e no ano. Vitória, ES, Brasil, 2019

\begin{tabular}{|c|c|c|c|c|c|c|c|c|c|}
\hline \multirow{3}{*}{ Variáveis } & \multicolumn{3}{|c|}{ Na vida } & \multicolumn{3}{|c|}{ No ano } & \multicolumn{3}{|c|}{ No mês } \\
\hline & \multicolumn{2}{|c|}{ Sim } & \multirow{2}{*}{$\mathrm{p}$-valor } & \multicolumn{2}{|c|}{ Sim } & \multirow{2}{*}{ p-valor } & \multicolumn{2}{|c|}{ Sim } & \multirow{2}{*}{ p-valor } \\
\hline & $n$ & $\%$ & & $n$ & $\%$ & & $\mathrm{n}$ & $\%$ & \\
\hline \multicolumn{10}{|l|}{ Sexo } \\
\hline Masculino & 5 & 4,9 & $0,362^{*}$ & 4 & 5,5 & $0,729^{*}$ & 2 & 3,4 & $0,291^{*}$ \\
\hline Feminino & 98 & 95,1 & & 69 & 94,5 & & 57 & 96,6 & \\
\hline \multicolumn{10}{|l|}{ Estado civil } \\
\hline $\begin{array}{l}\text { Solteiro / Separado / } \\
\text { Divorciado }\end{array}$ & 99 & 96,1 & $1,000^{*}$ & 71 & 97,3 & $0,653^{*}$ & 57 & 96,6 & $1,000^{*}$ \\
\hline Casado / Vive junto & 4 & 3,9 & & 2 & 2,7 & & 2 & 3,4 & \\
\hline
\end{tabular}




\begin{tabular}{|c|c|c|c|c|c|c|c|c|c|}
\hline \multirow{3}{*}{ Variáveis } & \multicolumn{3}{|c|}{ Na vida } & \multicolumn{3}{|c|}{ No ano } & \multicolumn{3}{|c|}{ No mês } \\
\hline & \multicolumn{2}{|c|}{ Sim } & \multirow{2}{*}{ p-valor } & \multicolumn{2}{|c|}{ Sim } & \multirow{2}{*}{ p-valor } & \multicolumn{2}{|c|}{ Sim } & \multirow{2}{*}{ p-valo } \\
\hline & $\mathbf{n}$ & $\%$ & & $n$ & $\%$ & & $\mathbf{n}$ & $\%$ & \\
\hline \multicolumn{10}{|l|}{ Grupo étnico } \\
\hline Branco & 45 & 43,7 & $0,448^{\dagger}$ & 29 & 39,7 & $0,143^{\dagger}$ & 24 & 40,7 & $0,326^{\dagger}$ \\
\hline Negro / Mulato / Pardo & 58 & 56,3 & & 44 & 60,3 & & 35 & 59,3 & \\
\hline \multicolumn{10}{|l|}{ Religião } \\
\hline Não tenho religião & 17 & 16,5 & $0,119^{*}$ & 14 & 19,2 & $0,046^{\dagger}$ & 13 & 22,0 & $0,014^{\dagger}$ \\
\hline Tenho religião & 86 & 83,5 & & 59 & 80,8 & & 46 & 78,0 & \\
\hline \multicolumn{10}{|l|}{ Prática religiosa } \\
\hline Sim & 76 & 73,8 & $0,037^{\dagger}$ & 52 & 71,2 & $0,045^{\dagger}$ & 41 & 69,5 & $0,041^{\dagger}$ \\
\hline Não & 27 & 26,2 & & 21 & 28,8 & & 18 & 30,5 & \\
\hline \multicolumn{10}{|l|}{ Você tem filhos? } \\
\hline Sim & 2 & 1,9 & $0,506^{*}$ & 1 & 1,4 & $0,581^{*}$ & 1 & 1,7 & $1,000^{*}$ \\
\hline Não & 101 & 98,1 & & 72 & 98,6 & & 58 & 98,3 & \\
\hline \multicolumn{10}{|l|}{ Reside com quem? } \\
\hline $\begin{array}{l}\text { Sozinho / Amigos / } \\
\text { Moradia estudantil / Outros }\end{array}$ & 27 & 26,2 & $0,976^{\dagger}$ & 19 & 26,0 & $0,970^{\dagger}$ & 18 & 30,5 & $0,303^{\dagger}$ \\
\hline $\begin{array}{l}\text { Pais / Cônjuge / Namorado } \\
\text { / Filhos }\end{array}$ & 76 & 73,8 & & 54 & 74,0 & & 41 & 69,5 & \\
\hline \multicolumn{10}{|l|}{ Atividade remunerada } \\
\hline Sim & 20 & 19,4 & $0,405^{*}$ & 11 & 15,1 & $0,375^{\dagger}$ & 10 & 16,9 & $0,840^{\dagger}$ \\
\hline Não & 83 & 80,6 & & 62 & 84,9 & & 49 & 83,1 & \\
\hline \multicolumn{10}{|l|}{ Classe Econômica } \\
\hline Classe A/B & 46 & 44,7 & $0,158^{\dagger}$ & 34 & 46,6 & $0,187^{\dagger}$ & 29 & 49,2 & $0,108^{\dagger}$ \\
\hline Classe C/D & 57 & 55,3 & & 39 & 53,4 & & 30 & 50,8 & \\
\hline Total & 103 & 100,0 & - & 73 & 100,0 & - & 59 & 100,0 & - \\
\hline
\end{tabular}

*Teste Exato de Fisher; ${ }^{\dagger}$ Teste do Qui-quadrado

Tabela 4 - Fatores associados ao uso de tabaco entre estudantes de Fonoaudiologia de uma universidade pública, segundo o uso na vida, no mês e no ano. Vitória, ES, Brasil, 2019

\begin{tabular}{|c|c|c|c|c|c|c|c|c|c|}
\hline \multirow{3}{*}{ Variáveis } & \multicolumn{3}{|c|}{ Na vida } & \multicolumn{3}{|c|}{ No ano } & \multicolumn{3}{|c|}{ No mês } \\
\hline & \multicolumn{2}{|c|}{ Sim } & \multirow{2}{*}{ p-valor } & \multicolumn{2}{|c|}{ Sim } & \multirow{2}{*}{ p-valor } & \multicolumn{2}{|c|}{ Sim } & \multirow{2}{*}{ p-valor } \\
\hline & $\mathrm{n}$ & $\%$ & & $n$ & $\%$ & & $\mathrm{n}$ & $\%$ & \\
\hline \multicolumn{10}{|l|}{ Sexo } \\
\hline Masculino & 4 & 14,3 & $0,065^{*}$ & 1 & 9,1 & $0,517^{*}$ & 1 & 10,0 & $0,483^{*}$ \\
\hline Feminino & 24 & 85,7 & & 10 & 90,9 & & 9 & 90,0 & \\
\hline \multicolumn{10}{|l|}{ Estado civil } \\
\hline $\begin{array}{l}\text { Solteiro / Separado / } \\
\text { Divorciado }\end{array}$ & 28 & 100,0 & $0,584^{*}$ & 11 & 100,0 & $1,000^{*}$ & 10 & 100,0 & $1,000^{*}$ \\
\hline Casado / Vive junto & - & - & & - & - & & - & - & \\
\hline \multicolumn{10}{|l|}{ Grupo étnico } \\
\hline Branco & 11 & 39,3 & $0,464^{\dagger}$ & 8 & 72,7 & $0,066^{*}$ & 6 & 60,0 & $0,511^{*}$ \\
\hline Negro / Mulato / Pardo & 17 & 60,7 & & 3 & 27,3 & & 4 & 40,0 & \\
\hline \multicolumn{10}{|l|}{ Religião } \\
\hline Não tenho religião & 7 & 25,0 & $0,067^{*}$ & 3 & 27,3 & $0,180^{*}$ & 3 & 30,0 & $0,143^{*}$ \\
\hline Tenho religião & 21 & 75,0 & & 8 & 72,7 & & 7 & 70,0 & \\
\hline \multicolumn{10}{|l|}{ Prática religiosa } \\
\hline $\operatorname{Sim}$ & 17 & 60,7 & $0,015^{\dagger}$ & 5 & 45,5 & $0,015^{*}$ & 4 & 40,0 & $0,008^{*}$ \\
\hline Não & 11 & 39,3 & & 6 & 54,5 & & 6 & 60,0 & \\
\hline \multicolumn{10}{|l|}{ Você tem filhos? } \\
\hline $\operatorname{Sim}$ & 1 & 3,6 & $0,520^{*}$ & - & - & $1,000^{*}$ & - & - & $1,000^{*}$ \\
\hline Não & 27 & 96,4 & & 11 & 100,0 & & 10 & 100,0 & \\
\hline
\end{tabular}




\begin{tabular}{|c|c|c|c|c|c|c|c|c|c|}
\hline \multirow{3}{*}{ Variáveis } & \multicolumn{3}{|c|}{ Na vida } & \multicolumn{3}{|c|}{ No ano } & \multicolumn{3}{|c|}{ No mês } \\
\hline & \multicolumn{2}{|c|}{ Sim } & \multirow{2}{*}{ p-valor } & \multicolumn{2}{|c|}{ Sim } & \multirow{2}{*}{ p-valor } & \multicolumn{2}{|c|}{ Sim } & \multirow{2}{*}{ p-valor } \\
\hline & $\mathrm{n}$ & $\%$ & & $n$ & $\%$ & & $\mathrm{n}$ & $\%$ & \\
\hline \multicolumn{10}{|l|}{ Reside com quem? } \\
\hline $\begin{array}{l}\text { Sozinho / Amigos / Moradia } \\
\text { estudantil / Outros }\end{array}$ & 7 & 25,0 & $0,875^{\dagger}$ & 3 & 27,3 & $1,000^{*}$ & 2 & 20,0 & $1,000^{*}$ \\
\hline $\begin{array}{l}\text { Pais / Cônjuge / Namorado } \\
\text { / Filhos }\end{array}$ & 21 & 75,0 & & 8 & 72,7 & & 8 & 80,0 & \\
\hline \multicolumn{10}{|l|}{ Atividade remunerada } \\
\hline Sim & 7 & 25,0 & $0,270^{*}$ & 2 & 18,2 & $1,000^{*}$ & 2 & 20,0 & $1,000^{*}$ \\
\hline Não & 21 & 75,0 & & 9 & 81,8 & & 8 & 80,0 & \\
\hline \multicolumn{10}{|l|}{ Classe Econômica } \\
\hline Classe A/B & 11 & 39,3 & $0,785^{\dagger}$ & 5 & 45,5 & $1,000^{*}$ & 5 & 50,0 & $0,740^{*}$ \\
\hline Classe C/D & 17 & 60,7 & & 6 & 54,5 & & 5 & 50,0 & \\
\hline Total & 28 & 100,0 & - & 11 & 100,0 & - & 10 & 100,0 & - \\
\hline
\end{tabular}

*Teste Exato de Fisher; ${ }^{+}$Teste do Qui-quadrado

A análise multivariada foi feita pela regressão logística binomial, tendo como categoria de referência o "Uso de álcool na vida, no ano e no mês" e "Uso de tabaco e derivados na vida, no ano e no mês", e incluiu todas as variáveis associadas ao resultado com p < 0,100 na análise univariada. Os resultados das análises multivariadas (Tabela 5) mostraram que, para as variáveis socioeconômicas e demográficas, a variável "idade" apresentou associação com a realização de "Uso de álcool no mês" após a inclusão na análise multivariada $\left[\left(\mathrm{OR}_{\text {bruto }}=\right.\right.$ $0,096)$ e $\left.\left(O R_{\text {ajustado }}=0,044\right)\right]$.

$\mathrm{Na}$ Tabela 5, a prática religiosa apresentou associação com a variável desfecho "Uso de álcool no mês" $\left[\left(\mathrm{OR}_{\text {bruto }}=0,044\right)\right.$ e $\left.\left(\mathrm{OR}_{\text {ajustado }}=0,020\right)\right]$, as variáveis "sexo" e "idade" apresentaram associação significativa com o "uso na vida de tabaco e derivados" na análise bivariada. Porém, a associação não se manteve após a análise multivariada.

A prática religiosa também se apresentou associada ao "uso no ano de tabaco e derivados" na análise bivariada, associação essa que também se manteve após a análise multivariada, "Uso de tabaco na vida" $\left[\left(\mathrm{OR}_{\text {bruto }}=0,018\right)\right.$ e $\left.\left(\mathrm{OR}_{\text {ajustado }}=0,026\right)\right]$, "Uso de tabaco no ano" $\left[\left(\mathrm{OR}_{\text {bruto }}=0,013\right)\right.$ e $\left.\left(\mathrm{OR}_{\text {ajustado }}=0,018\right)\right]$ (Tabela 5$)$.

Tabela 5 - Fatores associados ao uso (na vida, no mês e no ano) de álcool e tabaco entre estudantes da Fonoaudiologia de uma universidade pública. Vitória, ES, Brasil, 2019

\begin{tabular}{|c|c|c|c|c|c|c|c|c|c|c|c|c|}
\hline \multicolumn{13}{|c|}{ Uso de Álcool } \\
\hline \multirow[b]{2}{*}{ Variáveis* } & \multicolumn{4}{|c|}{ Na vida } & \multicolumn{4}{|c|}{ No ano } & \multicolumn{4}{|c|}{ No mês } \\
\hline & $\begin{array}{l}\text { ORbruto } \\
\text { (IC 95\%) }\end{array}$ & p-valor & $\begin{array}{c}\text { ORajustado } \\
\text { (IC } 95 \%)\end{array}$ & p-valor & $\begin{array}{l}\text { ORbruto } \\
\text { (IC 95\%) }\end{array}$ & p-valor & $\begin{array}{c}\text { ORajustado } \\
\text { (IC 95\%) }\end{array}$ & p-valor & $\begin{array}{l}\text { ORbruto } \\
\text { (IC 95\%) }\end{array}$ & p-valor & $\begin{array}{l}\text { ORajustado } \\
\text { (IC 95\%) }\end{array}$ & p-valor \\
\hline Idade & $\dagger$ & $\dagger$ & $\dagger$ & $\dagger$ & $\dagger$ & $\dagger$ & $\dagger$ & $\dagger$ & $\begin{array}{c}0,904 \\
(0,802- \\
1,018)\end{array}$ & 0,096 & $\begin{array}{c}0,875 \\
(0,768- \\
0,996)\end{array}$ & 0,044 \\
\hline $\begin{array}{l}\text { Prática } \\
\text { religiosa }\end{array}$ & $\dagger$ & $\dagger$ & $\dagger$ & $\dagger$ & & & $\dagger$ & $\dagger$ & & & & \\
\hline Sim & $\dagger$ & $\dagger$ & $\dagger$ & $\dagger$ & $\begin{array}{c}0,404 \\
(0,164- \\
0,997)\end{array}$ & 0,049 & $\dagger$ & $\dagger$ & $\begin{array}{c}0,418 \\
(0,179- \\
0,976)\end{array}$ & 0,044 & $\begin{array}{c}0,341 \\
(0,138- \\
0,842)\end{array}$ & 0,020 \\
\hline Não & $\dagger$ & $\dagger$ & $\dagger$ & $\dagger$ & & & $\dagger$ & $\dagger$ & & & & \\
\hline \multicolumn{13}{|c|}{ Uso de tabaco } \\
\hline & \multicolumn{4}{|c|}{ Na vida } & \multicolumn{4}{|c|}{ No ano } & \multicolumn{4}{|c|}{ No mês } \\
\hline Variáveis & $\begin{array}{l}\text { ORbruto } \\
\text { (IC 95\%) }\end{array}$ & $\mathrm{p}$-valor & $\begin{array}{l}\text { ORajustado } \\
\text { (IC 95\%) }\end{array}$ & $\mathrm{p}$-valor & $\begin{array}{l}\text { ORbruto } \\
\text { (IC 95\%) }\end{array}$ & $\mathrm{p}$-valor & $\begin{array}{l}\text { ORajustado } \\
\text { (IC 95\%) }\end{array}$ & $\mathrm{p}$-valor & $\begin{array}{l}\text { ORbruto } \\
\text { (IC 95\%) }\end{array}$ & p-valor & $\begin{array}{l}\text { ORajustado } \\
\text { (IC 95\%) }\end{array}$ & $\mathrm{p}$-valor \\
\hline Sexo & & & & & $\dagger$ & $\dagger$ & $\dagger$ & $\dagger$ & $\dagger$ & $\dagger$ & $\dagger$ & $\dagger$ \\
\hline Masculino & $\begin{array}{c}4,083 \\
(0,952- \\
17,513)\end{array}$ & 0,058 & $\begin{array}{c}3,662 \\
(0,815- \\
16,444)\end{array}$ & 0,090 & $\dagger$ & $\dagger$ & $\dagger$ & $\dagger$ & $\dagger$ & $\dagger$ & $\dagger$ & $\dagger$ \\
\hline Feminino & & & & & $\dagger$ & $\dagger$ & $\dagger$ & $\dagger$ & $\dagger$ & $\dagger$ & $\dagger$ & $\dagger$ \\
\hline
\end{tabular}




\begin{tabular}{|c|c|c|c|c|c|c|c|c|c|c|c|c|}
\hline $\begin{array}{l}\text { Grupo } \\
\text { étnico }\end{array}$ & $\dagger$ & $\dagger$ & $\dagger$ & $\dagger$ & & & & & $\dagger$ & $\dagger$ & $\dagger$ & $\dagger$ \\
\hline Branco & $\dagger$ & $\dagger$ & $\dagger$ & $\dagger$ & & & & & $\dagger$ & $\dagger$ & $\dagger$ & $\dagger$ \\
\hline $\begin{array}{l}\text { Negro / } \\
\text { Mulato / } \\
\text { Pardo }\end{array}$ & $\dagger$ & $\dagger$ & $\dagger$ & $\dagger$ & $\begin{array}{c}0,281 \\
(0,071- \\
1,113)\end{array}$ & 0,071 & $\begin{array}{c}0,296 \\
(0,073-1,209)\end{array}$ & 0,090 & $\dagger$ & $\dagger$ & $\dagger$ & $\dagger$ \\
\hline $\begin{array}{l}\text { Prática } \\
\text { religiosa }\end{array}$ & & & & & & & & & $t$ & $\dagger$ & $\dagger$ & $\dagger$ \\
\hline Sim & $\begin{array}{c}0,331 \\
(0,133- \\
0,826)\end{array}$ & 0,018 & $\begin{array}{c}0,348 \\
(0,137- \\
0,882)\end{array}$ & 0,026 & $\begin{array}{c}0,200 \\
(0,056- \\
0,712)\end{array}$ & 0,013 & $\begin{array}{c}0,209 \\
(0,057-0,760)\end{array}$ & 0,018 & $t$ & $\dagger$ & $\dagger$ & $\dagger$ \\
\hline Não & & & & & & & & & $\dagger$ & $\dagger$ & $\dagger$ & $\dagger$ \\
\hline
\end{tabular}

*Somente variáveis que apresentaram associação menor ou igual a 10\% no modelo de regressão logística; 'Variáveis que não foram incluídas no modelo de regressão logística ou que não mantiveram associação significativa com a variável desfecho correspondente no modelo de regressão logística

\section{Discussão}

Cuidar da saúde engloba não apenas o cuidado com as pessoas, mas principalmente a atenção à própria saúde. A saúde está ligada a fatores como a qualidade de vida. O reconhecimento de que o álcool e o tabaco causam influências danosas foi definido como problema de saúde pública mundial(12).

O aumento do consumo do álcool e tabaco entre os jovens está evidenciado em pesquisas recentes ${ }^{(5,6-8)}$. Elevadas prevalências de consumo de álcool foram encontradas no estudo epidemiológico brasileiro e variaram de $66,3 \%$ a $91,9 \%{ }^{(12)}$. De acordo com o Centro Brasileiro de Informações sobre Drogas Psicotrópicas (CEBRID), 73,2\% dos jovens entre 18 e 24 anos já fizeram uso de bebidas alcoólicas alguma vez na vida e 15,5\% apresentam sintomas de dependência(7-8,13).

Entre os principais resultados, foi identificado o uso de álcool entre o universitário nos últimos 30 dias, 69\% fizeram uso de bebidas alcoólicas, enquanto $51,1 \%$ dos não universitários consumiram álcool nos últimos 30 dias. O uso de álcool pelos universitários também superou o consumo identificado entre os não universitários para uso na vida e nos últimos 12 meses $^{(14)}$.

Nos dados encontrados no I Levantamento Nacional sobre o Uso de Álcool e Outras Drogas entre universitários das 27 capitais brasileiras, observou-se que $86,2 \%$ dos universitários já fizeram uso de álcool alguma vez na vida e $72 \%$, no ano. Em relação ao uso de tabaco, identificou-se o uso na vida de $46,7 \%$ e no ano de $27,8 \%$. A prevalência do uso das substâncias lícitas nos últimos 30 dias que antecederam a pesquisa foram: álcool $60,5 \%$ e tabaco $21,6 \%{ }^{(10)}$.

Em relação ao sexo, o II Levantamento Nacional de Álcool e Drogas comparou resultados do consumo de álcool entre homens e mulheres em dois períodos, 2006 e 2012. No ano de 2006, mulheres que apresentavam uso regular (uma ou mais vezes por semana) de consumo de álcool representaram 27\%, aumentando para 38\% em 2012, enquanto que, em relação aos homens, houve aumento de $54 \%$ para $63 \%$; a pesquisa concluiu que mulheres, especialmente as mais jovens, são a população mais vulnerável aos riscos, já que apresentaram maiores índices, bebendo em binge ${ }^{(15)}$.

Uma pesquisa realizada em 2015, no Brasil, III Levantamento Nacional sobre o uso de drogas pela população brasileira, com pessoas de idade entre os 12 e os 65 anos, teve como objetivo estimar e avaliar os parâmetros epidemiológicos do uso de drogas. Os resultados revelaram que mais de um milhão de adolescentes e jovens, na faixa etária dos 18 aos 34 anos, consumiram álcool e tabaco nos doze meses anteriores à pesquisa(6).

De acordo com o presente estudo, o consumo de álcool foi elevado entre os acadêmicos entrevistados, $79,2 \%$ relataram ter feito uso de álcool e $21,5 \%$ de tabaco em algum momento na vida, resultado semelhante foi observado em uma pesquisa com universitários da área da saúde em que a prevalência do consumo de álcool pelos estudantes, pelo menos uma vez na vida, foi de $79,7 \% \%^{(13)}$.

Em relação aos padrões de uso de álcool pela população estudada, os dados encontrados neste estudo corroboram os encontrados em outras pesquisas ${ }^{(16-17)}$. A prevalência do uso de álcool nos últimos 30 dias que antecederam a pesquisa corrobora estudo também realizado entre universitários de Terapia Ocupacional de uma universidade pública(16).

Pesquisas realizadas na Universidade Federal do Maranhão constataram que os estudantes que faziam uso de álcool representaram $64,5 \%$, na faculdade de medicina do sul de Minas Gerais, constatou-se que $99 \%$ dos estudantes já consumiram bebida alcoólica uma vez na vida e, na pesquisa realizada entre estudantes da área da saúde da Universidade Estadual de Montes Claros, $74,9 \%$ relataram ter feito uso de álcool no último ano ${ }^{(1,18)}$.

No estudo realizado entre estudantes do curso de nutrição de uma universidade de Curitiba, 78,1\% dos estudantes relataram já ter feito uso de álcool na vida, o resultado encontrado corrobora os dados do presente estudo ${ }^{(19)}$. Nos resultados mostrados no estudo realizado entre estudantes do curso de enfermagem da Universidade de Passo Fundo, o uso de álcool na vida foi de $93,6 \%$, em relação ao uso no ano $89,8 \%$ e no mês $78,9 \%{ }^{(20)}$. 
Na pesquisa realizada na Universidade Federal do Espírito Santo (UFES), nos cursos de Farmácia, Odontologia e Psicologia, observou-se maior prevalência de uso álcool e tabaco na vida. Para os três cursos, foram encontrados valores de $86,5 \%, 87,9 \%$ e $85,07 \%$ para uso de álcool na vida, e $28,4 \%, 27 \%$ e $33,03 \%$ dos alunos relataram já ter usado o tabaco alguma vez na vida, respectivamente(21-23).

Entre os jovens, as possibilidades de vivenciar esses problemas são maiores, uma vez que identificamos a exposição precoce a essas substâncias ${ }^{(7)}$. Estima-se que o uso do álcool esteja aumentado em razão de estilo de vida, ansiedade, estresse, depressão e baixa autoestima. Os jovens têm cultivado o hábito de se embriagar. A dependência alcoólica é um fator preocupante que pode ser intensificado com a associação ao tabaco(5).

Importante evidenciar que o consumo do álcool pode ser o gatilho inicial para o uso de outras drogas como o tabaco, que é a segunda droga mais consumida entre os estudantes ${ }^{(1)}$. Alguns dos fatores que podem estar associados ao uso do tabaco entre os universitários, principalmente na área da saúde, são a curiosidade, imitação e aceitação social. A prevalência do uso de tabaco entre estudantes da área da saúde foi entre $15 \%$ e $22 \%$, vale ressaltar que possuem alguns fatores de risco para o fumo, como a presença de outros familiares fumantes, morar sozinho, mau desempenho acadêmico, uso prévio de álcool(1).

Desde a década passada, já se notava que hábitos adquiridos na vida universitária poderiam ser levados para a vida profissional(5). Esses fatos e dados requerem atenção especial, pois, os estudantes atuais serão futuros profissionais que vão transmitir conhecimento sobre os cuidados à saúde, e muitos serão modelos de conduta. Ainda que o consumo de álcool e tabaco no mês, no ano e na vida pelos estudantes de Fonoaudiologia seja menor que o encontrado na maioria dos estudos $\operatorname{citados}^{(2,21-23)}$, mesmo assim, o consumo é preocupante.

Neste estudo, pôde ser observada a associação da prática religiosa com o uso de álcool e tabaco, dados mostraram que os universitários que praticam a religião têm chances de proteção ao uso indevido de álcool e tabaco. A ausência da prática religiosa pode aumentar o consumo problemático do álcool(24-26). Resultados semelhantes também foram encontrados em outros estudos $^{(25-26)}$. De acordo com o levantamento feito nas 27 capitais brasileiras, $48 \%$ dos universitários sem religião já haviam experimentando alguma droga contra $33,2 \%$ dos que possuem religião(10).

$\mathrm{Na}$ pesquisa realizada entre os pacientes atendidos na Clínica Saúde da Família, no Rio de Janeiro, a presença da religião pareceu ter efeito protetor para o consumo de álcool e tabaco, reforçou a ideia de que ir à igreja ou a encontros religiosos distanciaria os indivíduos do consumo nocivo de álcool e tabaco(27). O fato de algumas religiões pregarem a abstinência alcoólica pode estar associado ao menor consumo da substância ${ }^{(28)}$.

Levando em consideração a relevância do uso de álcool e de tabaco entre os universitários, a identificação prévia poderá acudir com estratégias para prevenção de problemas causados por essas substâncias, sendo importante priorizar as ações de promoção de saúde, com ênfase na redução do consumo de álcool e tabaco entre os grupos mais vulneráveis, de forma a evitar a manutenção de tal padrão bem como sua evolução para casos de vícios.

A divulgação diante do uso dessas substâncias ocasiona, entre os jovens, um despertar para o estímulo do consumo e a criação de hábito. O reconhecimento dos riscos à exposição precoce e problemática, considerando que o uso do álcool e tabaco está associado a vários malefícios, ocasionando prejuízos à saúde e à vida desses universitários, torna-se necessário traçar medidas de promoção à saúde e prevenção ao uso(29).

Entre as limitações deste estudo, inclui-se seu delineamento transversal, pois não é possível estabelecer correlações entre o consumo de álcool e tabaco com as variáveis associadas a ele, bem como não podemos generalizar os resultados para toda a população. O uso de questionários facilita a omissão de informações, porém, por serem anônimos, deixam o participante com mais liberdade para responder a questões pessoais. Além disso, os estudos relacionados ao uso de substâncias lícitas entre os estudantes de Fonoaudiologia são raros e limitados. No entanto, os achados sobre associações aqui encontrados servem como ponto de partida para estudos futuros.

\section{Conclusão}

Os dados deste estudo demonstram um elevado consumo de substâncias lícitas entre os estudantes do curso de Fonoaudiologia de uma universidade pública. Estudos relacionados ao consumo de álcool e tabaco entre estudantes de Fonoaudiologia são escassos, são necessárias mais pesquisas para compreender, com nitidez, os resultados e as associações encontradas no presente trabalho.

Os resultados encontrados no estudo são similares aos de outras pesquisas com os universitários, revelando a necessidade da abordagem deste tema no currículo acadêmico, a fim de contribuir para a formação de profissionais de saúde preparados para a assistência de pacientes com problemas decorrentes de consumo do álcool e tabaco.

Os achados dão subsídios para a criação de ações, políticas e programas de prevenção do uso indevido de álcool e tabaco entre universitários. Uma forma de prevenção, além de políticas e programas, seria a inclusão e/ou ampliação do tema na matriz curricular. 


\section{Referências}

1. Gomes IP, Pereira RA da C, Santos BF dos, Pinheiro M de A, Alencar CH, Cavalcanti LP de G. Fatores Associados à Manutenção do Vício de Fumar e do Consumo de Álcool entre Acadêmicos de Medicina em uma Capital do Nordeste do Brasil. Rev Bras Educ Méd. 2019;43(1):55-64. doi: http://doi.org/10.1590/1981-52712015v43n1rb20180068 2. Portugal FB, Siqueira MM de. Fatores associados ao uso de substâncias psicoativas entre universitários de pedagogia da Universidade Federal do Espírito Santo. Cad Saúde Coletiva. 2011;19(3). doi: http://doi.org/10.1590/ S0047-20852008000300006

3. Peuker AC, Fogaça J, Bizarro L. Expectativas e beber problemático entre universitários. Psicol Teoria Pesqui. 2006;22(2):193-200. doi: http://doi.org/10.1590/ S0102-37722006000200009

4. Pereira ISSD, Melo RA, Gurgel AJP, Barbosa AGM, Zanatta IC, Melo SLA, et al. Uso de bebidas alcoólicas por estudantes de medicina. J Health NPEPS. 2020;5(1):24260. doi: http://doi.org/10.30681/252610104307

5. Pinheiro M de A, Torres LF, Bezerra MS, Cavalcante RC, Alencar RD, Donato AC, et al. Prevalência e Fatores Associados ao Consumo de Álcool e Tabaco entre Estudantes de Medicina no Nordeste do Brasil. Rev Bras Educ Méd. 2017;41(2):231-9. doi: http://doi. org/10.1590/1981-52712015v41n2rb20160033

6. Bastos FIPM, Vasconcellos MT, De Boni RB, Reis NB, Coutinho CFS. III Levantamento Nacional sobre o uso de drogas pela população brasileira. Rio de Janeiro: FIOCRUZ/ICICT; 2017

7. Magalhães L de SP de, Vernaglia TVC, Sousa FAM de, Chagas SV da, Cruz MS. The drugs phenomenon from the perspective of nursing students: patterns of consumption, attitudes and beliefs. Esc Anna Nery. 2018;22(1). doi: http://doi.org/10.1590/2177-9465-ean-2017-0205

8. Rodríguez-Muñoz PM, Carmona-Torres JM, RodríguezBorrego MA. Influence of tobacco, alcohol consumption, eating habits and physical activity in nursing students. Rev. Latino-Am. Enfermagem. 2020;28. doi: http://doi. org/10.1590/1518-8345.3198.3230

9. Moreira T de C, Gadenz C, Figueiró LR, Capobianco DM, Cunha K, Ferigolo M, et al. Uso de substâncias psicoativas, alterações vocais e qualidade de vida em usuários de drogas lícitas e ilícitas. Rev CEFAC. 2015;17(2):374-84. doi: http://doi.org/10.1590/1982 021620156714

10. Andrade AG, Duarte PCAV, Oliveira LG, organizadores. I Levantamento Nacional sobre o Uso de Álcool e Outras Drogas entre Universitários das 27 Capitais Brasileiras. Observatório Brasileiro de Informações sobre Drogas, Faculdade de Medicina da Universidade de São Paulo, Secretaria Nacional de Políticas sobre Drogas. Brasília; 2010. $284 \mathrm{p}$

11. Conselho Nacional de Saúde (BR). Resolução n. $196 / 96$ regulamenta e estabelece as diretrizes para as pesquisas envolvendo seres humanos no Brasil. Brasília; 1996. [Acesso 10 ago 2019]. Disponível em: http://www. conselho.saude.gov.br/

12. Henriquéz PC, Carvalho AMP de. Perceptions of drugs benefits and barriers to quit by undergraduate health students. Rev. Latino-Am. Enfermagem. 2008;16( n.spe). doi: http://doi.org/90/S0104-11692008000700019 13. Mendonça $A K R H$, Jesus CVF de, Figueiredo MBG de A, Valido DP, Nunes MAP, Lima SO. Alcohol consumption and factors associated with binge drinking among female university students of health area. Esc Anna Nery. 2018;22(1). doi: http://doi. org/10.1590/2177-9465-ean-2017-0096

14. Barros MSMR de, Costa LS. Perfil do consumo de álcool entre estudantes universitários. SMAD, Rev Eletrônica Saúde Mental Álcool Drog. 2019;15(1). doi: http://doi. org/10.11606/issn.1806-6976.smad.2019.000353

15. Laranjeira R, Madruga CS, Pinsky I, Caetano R, Ribeiro M, Mitsuhiro S. II Levantamento Nacional de Álcool e Drogas Consumo de Álcool no Brasil: Tendências entre 2006/2012. São Paulo: INPAD; 2013

16. Pinho MC de, Souza RCF de, Portugal FB, Siqueira MM de. Uso de álcool e tabaco entre universitários de Terapia Ocupacional de uma universidade pública. SMAD, Rev Eletrônica Saúde Mental Álcool Drog. (Ed. port.) 2020;16(1). doi: http://doi.org/10.11606/issn.18066976.smad.2020.152411

17. Santos DDM, Guimarães MM, Bodevan EC, Rocha RL, Pinheiro MLP. Uso de substâncias psicoativas entre estudantes universitários. SMAD, Rev Eletrônica Saúde Mental Álcool e Drog. 2019;15(3):1-9. doi: http://doi. org/10.11606/issn.1806-6976.smad.2019.148973

18. Pelicioli M. Perfil do consumo de álcool e prática do beber pesado episódico entre universitários brasileiros da área da saúde. J Bras Psiquiatria. 2017; 66(3):150-6. doi: http://doi.org/10.1590/0047-2085000000164

19. Chiapetti N, Serbena CA. Uso de álcool, tabaco e drogas por estudantes da área de saúde de uma Universidade de Curitiba. Psicol Reflexão Crítica. 2007;20(2). doi: http:// doi.org/10.1590/S0102- 79722007000200017

20. Picolotto E, Libardoni LFC, Migott AMB, Geib LTC. Prevalência e fatores associados com o consumo de substâncias psicoativas por acadêmicos de enfermagem da Universidade de Passo Fundo. Ciênc Saúde Coletiva. 2010;15(3):645-54. doi: http://doi.org/10.1590/ S1413-81232010000300006

21. Teixeira RF, Souza RS, Buaiz V, Siqueira MM. Uso de substâncias psicoativas entre estudantes de odontologia da Universidade Federal do Espírito Santo. Rev Ciênc Saúde Coletiva. 2010;15(3):655-62. doi: http://doi. org/10.1590/S1413-81232010000300007

22. Portugal FB, Souza RS, Buaiz V, Siqueira MM. Uso de drogas por estudantes de Farmácia da Universidade Federal 
do Espírito Santo. J Bras Pisquiatria. 2008;57(2):127-32. doi: http://doi.org/10.1590/ S0047-20852008000200008 23. Santos MVF, Pereira DS, Siqueira MM. Uso de álcool e tabaco entre estudantes de Psicologia da Universidade Federal do Espírito Santo. J Bras Psiquiatria. 2013;62(1):22-30. doi: http://doi.org/10.1590/ S0047-20852013000100004

24. Bastos FI, Bertoni N, Hacker MA. Consumo de álcool e drogas: principais achados de pesquisa de âmbito nacional, Brasil 2005. Rev Saúde Pública. 2008;42(suppl 1):109-17. doi: http://doi.org/10.1590/S0034-89102008000800013 25. Vargas D de, Oliveira MAF de, Araújo EC. Prevalência de dependência alcoólica em serviços de atenção primária à saúde de Bebedouro, São Paulo, Brasil. Cad Saúde Pública. 2009;25(8):1711-20. doi: http://doi. org/10.1590/S0102-311X2009000800007

26. Amato TDC, Silveira PS da, Oliveira JS de, Ronzani TM. Uso de bebida alcoólica, religião e outras características sociodemográficas em pacientes da atenção primária à saúde - Juiz de Fora, MG, Brasil - 2006. SMAD, Rev Eletrônica Saúde Mental Álcool e Drog. 2008;4(2):1. doi: http://doi.org/10.11606/issn.1806-6976.v4i2p01-17

27. Queiroz N da R, Portella LF, Abreu AMM. Associação entre o consumo de bebidas alcoólicas e tabaco e a religiosidade. Acta Paul Enferm. 2015;28(6):546-52. doi: http://doi.org/10.1590/1982-0194201500091

28. Jomar RT, Abreu ÂMM, Griep RH. Padrões de consumo de álcool e fatores associados entre adultos usuários de serviço de atenção básica do Rio de Janeiro, RJ, Brasil. Cienc Saúde Coletiva. 2014;19(1):27-37. doi: http://doi. org/10.1590/1413-81232014191.2009

29. Malta DC, Mascarenhas MDM, Porto DL, Duarte EA, Sardinha LM, Barreto SM, et al. Prevalência do consumo de álcool e drogas entre adolescentes: análise dos dados da Pesquisa Nacional de Saúde Escolar. Rev Bras Epidemiol. 2011;14(suppl 1):136-46. doi: http://doi.org/10.1590/ S1415-790X2011000500014

\section{Contribuição dos autores}

Concepção e planejamento do estudo: Nathalia Gama Puppim, Flávia Batista Portugal, Marluce Mechelli de Siqueira. Obtenção dos dados: Nathalia Gama Puppim, Marluce Mechelli de Siqueira. Análise e interpretação dos dados: Nathalia Gama Puppim, Flávia Batista Portugal, Marluce Mechelli de Siqueira. Análise estatística: Nathalia Gama Puppim, Flávia Batista Portugal, Marluce Mechelli de Siqueira. Redação do manuscrito: Nathalia Gama Puppim, Marluce Mechelli de Siqueira. Revisão crítica do manuscrito: Nathalia Gama Puppim, Marluce Mechelli de Siqueira.

Todos os autores aprovaram a versão final do texto.

Conflito de interesse: os autores declararam que não há conflito de interesse. 